\title{
The relationship between body mass index and health-related quality of life in urban disadvantaged children
}

\author{
Ciara Wynne - Catherine Comiskey • \\ Eleanor Hollywood • Mary Brigid Quirke • \\ Karin O'Sullivan · Sinéad McGilloway
}

Accepted: 21 January 2014/Published online: 29 January 2014

(c) Springer International Publishing Switzerland 2014

\begin{abstract}
Introduction The study's aim was to establish, for children living in urban disadvantage, the nature and extent of the relationship between body mass index (BMI) and healthrelated quality of life (HRQoL), including the role of individual and family factors in influencing this relationship.

Methods Within the context of a longitudinal design, 255 children aged 7-12 years (50\% male) self-reported their HRQoL (Kidscreen-27) and had their height and weight measured at year one and two. One parent/guardian for each child was also assessed at year one with the OSLO Social Support Scale and Hospital Anxiety and Depression Scale. Regression analysis was also conducted.

Results BMI was weakly inversely associated with 'total HRQoL' $(r=-.15, p<.05)$, 'physical well-being' and 'autonomy and parent relations'. Significant differences were found between normal weight and obese children on all but the latter dimension. Neither weight group, however, fell below the average European HRQoL range. BMI predicted physical well-being a year later and vice versa, whilst autonomy and parent relations also predicted BMI a year later. In terms of 'overweight' children (38 \%), those approaching adolescence had poorer physical and school well-being than younger children, and those whose parents had moderate-to-severe levels of depression fared worse on
\end{abstract}

Electronic supplementary material The online version of this article (doi:10.1007/s11136-014-0634-7) contains supplementary material, which is available to authorized users.

C. Wynne $(\bowtie) \cdot$ C. Comiskey · E. Hollywood · K. O’Sullivan Trinity College Dublin, Dublin, Ireland

e-mail: wynnec1@tcd.ie

M. B. Quirke · S. McGilloway

National University of Ireland Maynooth, Kildare, Ireland school well-being than children whose parents were not depressed.

Conclusion The findings suggest that obesity programmes could aim to prevent/reduce obesity and optimise HRQoL in urban disadvantaged preadolescent children whilst also targeting parental mental health difficulties. Future research should examine mediators of the effect of BMI on HRQoL.

Keywords Health-related quality of life - Body mass index · Obesity · Children · Disadvantage · Urban . Moderation

\section{Introduction}

Obesity is now recognised as the most prevalent childhood disease worldwide [1]. In Ireland, one in four children is overweight [2]. Childhood obesity can have adverse psychosocial as well as medical consequences $[3,4]$ such as reduced health-related quality of life (HRQoL). HRQoL refers to a subjective evaluation of the quality of multiple life dimensions in terms of health [5] that is also affected by a complex interaction of other person factors, processes and contexts [6,7]. For obese children from either clinical or general populations, the HRQoL dimensions of physical and social well-being are particularly affected [8-10]. The HRQoL of overweight children can also be negatively affected [i.e. the risk of HRQoL increases as body mass index (BMI) scores increase] [11, 12]. Notably, few studies have explored the risk to HRQoL over time [13, 14].

The risk of obesity $[15,16]$ and poor HRQoL [17] is much greater for children living in poverty such as those in densely populated urban disadvantaged areas. According to the family 'investment' and 'stress' models [18], a low income can adversely affect children's health because it 
hinders parents' ability to purchase helpful materials, experiences and services [19, 20]. It can also negatively impact parental mental health [21], which can lead to dysfunctional parenting practices and in turn, poorer health outcomes for children [22]. However, we were unable to locate any studies, to date, which have explored the effect of BMI on the HRQoL of urban disadvantaged children.

Few studies have examined the factors that intensify or reduce the effect of obesity on children's HRQoL (i.e. moderator effects analysis) [11]. For example, there has been little or no research investigating the extent to which the negative effect of overweight BMI on children's HRQoL can be modified by the age and gender of the child or their parent's mental health and social support. Studies indicate that overweight adolescents and girls may be more likely to experience lower HRQoL than overweight children and boys [23, 24]. Indirect evidence suggests that parental mental ill health may also increase the risk of being overweight on children's HRQoL and that parents' social support may protect the HRQoL of overweight children. More specifically, children whose parents have mental health difficulties, particularly depression, are more likely to be overweight $[25,26]$ and to have poor HRQoL [27] than children whose parents have positive mental health. In addition, children whose parents have strong social support available to them (or living in a two-parent family) are less likely to be overweight $[28,29]$ and to have impaired HRQoL [30, 31] than their 'low social support' counterparts.

The objectives of this study were to determine in a sample of urban disadvantaged children: (1) whether BMI significantly negatively correlates with HRQoL; (2) between what weight groups do significant differences occur; (3) whether BMI predicts HRQoL a year later; and under conditions of high BMI, (4) whether those approaching adolescence, who are female or whose parents have high depressive symptoms, have a lower HRQoL than their counterparts; and (5) whether those whose parents have strong perceived social support or who live in a two-parent family have better HRQoL than their counterparts.

\section{Methods}

\section{Sample}

The study involved a prospective longitudinal cohort design. A total of 255 children aged 7-12 years $(50 \%$ male) were recruited using consecutive sampling across seven schools in highly disadvantaged urban districts located in the Greater Dublin Area in Ireland (according to the Government [32] and the Deprivation Index [33]). One of their parents/guardians $(N=219,90 \%$ mothers $)$ was also invited to participate in the study. Children's HRQoL, height and weight were assessed in school at year one and two. In addition, parents were administered a battery of measures over the phone at year one. Institutional ethical approval was obtained for this study.

\section{Measures}

Children's HRQoL was assessed using the Kidscreen-27, a generic 27-item self-report for children aged 8-18 years [34]. Using a five-point Likert scale, it assesses five dimensions of well-being: (1) physical well-being, (2) psychological well-being, (3) autonomy and parent relations, (4) social support and peer relations and (5) school environment. The t-score from each dimension ranges from 0 to 100 , with a mean of $50(\mathrm{SD}=10)$. Higher scores indicate better HRQoL. Items within the Kidscreen-10 were extracted from the Kidscreen-27 to calculate a total HRQoL score. The Kidscreen-27 and Kidscreen-10 are psychometrically robust with Cronbach's $\alpha$ satisfactorily over .73 [34-36]. Cronbach's alpha in the present study was satisfactory at over .73 .

Children's body fat was determined by their BMI [weight/ (height $\left.)^{2}\right]$ as measured by children's nurses trained in anthropometric measurement [15]. The World Health Organisation's (WHO) AnthroPlus software [37] converted BMIs into age- and gender-specific standardised $z$-scores ranging between -3 and 3 and identified children's weight category using WHO (2007) thresholds for overweight (i.e. +1 SD or $25 \mathrm{~kg} / \mathrm{m}^{2}$ ) and obesity (i.e. $+2 \mathrm{SD}$ or $30 \mathrm{~kg} / \mathrm{m}^{2}$ ) [38].

The depressive symptoms of parents were measured with the Hospital Anxiety and Depression Scale (HADS), a brief 14-item self-report clinical objective measure of depressive and anxiety symptoms for adults over 16 years [39]. The HADS takes less than $10 \mathrm{~min}$ to complete and has been used widely in research [40] and in clinical practice [41]. Normative data from large non-clinical samples have been published [42, 43]. Each dimension has a cumulative total score between 0 and 21. Raw scores between 11 and 15 identify moderate cases, whilst scores of 16 or above identify severe cases [44]. The measure has good psychometric properties [45] with a Cronbach's $\alpha$ greater than .80 [46]. In the present study, $\alpha$ was .97 .

The parent's perceived social support was assessed using the three-item Oslo Social Support self-report Scale [47]. Responses provided a cumulative total score (3-14 points) that reflected 'poor' (3-8), 'moderate' (9-11) or 'strong' (12-14) levels of social support. The measure has good psychometric properties [47] with Cronbach's $\alpha$ over .72 [48]. Cronbach's alpha in this current study was below satisfactory levels at .63 , but closer examination of the results (i.e. corrected item-total correlation and Cronbach's $\alpha$ if item deleted) revealed that the items were measuring the same thing as the scale. Family structure was recorded within a profile questionnaire. 
Statistical analysis

Pearson's $r$ correlations were used to test the bivariate association between continuous variables, whilst the distributions of HRQoL were compared between the groups of categorical variables with two or more than two levels using independent $t$ tests and one-way analysis of variance (ANOVA). Confounders were also explored. Linear regression determined whether BMI predicted HRQoL a year later. In order to determine whether five variables (gender, age, depressive symptoms, perceived social support and family structure) moderated the effect of BMI on HRQoL, HRQoL was regressed onto (a) BMI; (b) the potential moderator; and c) the product of BMI and the potential moderator using hierarchical multiple regression (HMR) analysis [49]. Simple slope analyses were used to examine the direction and degree of moderation found, and regression lines were graphed. All analyses were conducted with SPSS (version 18). Notably, some HRQoL distributions were skewed and non-normal. However, this is common as most people experience a positive HRQoL. It was estimated that between 78 and 558 children were needed to conduct the HMR based on the probability of type I error of 0.05 alpha, power of 0.8 , medium and small effect size $\left(f^{2}\right)$ of 0.15 and 0.02 , and attrition of $2 \%$ [50].

\section{Results}

Sample descriptives

Table 1 presents a description of the children and their parents/guardians at year one of the study. The mean HRQoL scores were within the average range for the general population of Irish and European children [34], which was unexpected as children from low socioeconomic status (SES) populations tend to have poorer HRQoL. School well-being was ranked highest, which contrasts with the general population of Irish children and children living in socioeconomic disadvantage across Europe, where it was ranked as lowest and second lowest, respectively $[17,51]$. Furthermore, over a third of children $(38 \%$, $96 / 253$ ) were considered either overweight (16\%) or obese (22\%), which is greater than the rate of $25 \%$ observed in a nationally representative sample [2] as well as the $33 \%$ rate in a socioeconomically disadvantaged sample [52] of Irish children.

Whilst a significant proportion of parents were single, most $(60 \%, 153 / 219)$ lived with their partner (i.e. another biological parent, step parent or foster parent). The ratio of two- to one-parent households in this study (60:40) was, as expected, lower than the ratio in Ireland more generally
Table 1 Description of the child and parent sample

\begin{tabular}{|c|c|c|}
\hline Variables & $N(\%)$ & $M(S D)$ range \\
\hline Child age & $255(100)$ & 9.73 (1.46) $7-12$ \\
\hline Child gender & $255(100)$ & \\
\hline Male & $128(50)$ & \\
\hline Female & $127(50)$ & \\
\hline Child ethnicity $^{\mathrm{a}}$ & $249(98)$ & \\
\hline Caucasian & $236(95)$ & \\
\hline Other & $13(5)$ & \\
\hline Child BMI z-scores & $253(99)$ & $\begin{array}{l}0.84(1.10)-2.21 \text { to } \\
\quad 4.41\end{array}$ \\
\hline Underweight & $1(.4)$ & \\
\hline Normal weight & $156(61.6)$ & \\
\hline Overweight & $41(16)$ & \\
\hline Obese & $55(22)$ & \\
\hline Child total HRQoL & $245(96)$ & $\begin{array}{l}53.86(12.0) \\
26.64-83.81\end{array}$ \\
\hline Below average total HRQoL & $43(18)$ & \\
\hline Average total HRQoL & $162(66)$ & \\
\hline Above average total HRQoL & $40(16)$ & \\
\hline Physical well-being & $253(99)$ & $\begin{array}{l}53.57(11.1) \\
20.70-73.20\end{array}$ \\
\hline Psychological well-being & $252(99)$ & $\begin{array}{l}53.56(10.9) \\
20.55-75.53\end{array}$ \\
\hline Autonomy and parent relations & $252(99)$ & $\begin{array}{l}52.07(12.9) \\
13.96-74.39\end{array}$ \\
\hline $\begin{array}{l}\text { Social support and peer } \\
\text { relations }\end{array}$ & $251(99)$ & $\begin{array}{l}53.26(13.3) \\
11.24-66.34\end{array}$ \\
\hline School environment & $251(99)$ & $\begin{array}{l}55.47(11.9) \\
16.28-71.00\end{array}$ \\
\hline Family structure & $255(100)$ & \\
\hline One-parent family & $102(40)$ & \\
\hline Two-parent family & $153(60)$ & \\
\hline $\begin{array}{l}\text { Guardian's relationship to } \\
\text { child }^{\mathrm{a}}\end{array}$ & $219(100)$ & \\
\hline Mother & $196(90)$ & \\
\hline Father & $18(8)$ & \\
\hline Other & $5(2)$ & \\
\hline Guardian age $^{\mathrm{a}}$ & $219(100)$ & 37.35 (7.29) 25-61 \\
\hline Guardian marital status $^{\mathrm{a}}$ & $219(100)$ & \\
\hline Single & $93(42)$ & \\
\hline Cohabiting & $50(23)$ & \\
\hline Married & $76(35)$ & \\
\hline $\begin{array}{l}\text { Guardian education } \\
\text { completed }^{\mathrm{a}}\end{array}$ & $219(100)$ & \\
\hline $\begin{array}{l}\text { Up to junior certificate } \\
\text { obtained }\end{array}$ & $102(47)$ & \\
\hline $\begin{array}{l}\text { Junior certificate and more } \\
\text { obtained }\end{array}$ & $117(53)$ & \\
\hline Guardian employment status ${ }^{\mathrm{a}}$ & $219(100)$ & \\
\hline Employed & $114(52)$ & \\
\hline Other & $105(48)$ & \\
\hline
\end{tabular}


Table 1 continued

\begin{tabular}{lrl}
\hline Variables & $N(\%)$ & $M(S D)$ range \\
\hline $\begin{array}{l}\text { Guardian perceived social } \\
\quad \text { support }\end{array}$ & $219(100)$ & $11.73(2.34) 4-14$ \\
Poor & $23(11)$ & \\
Moderate & $55(25)$ & \\
Strong & $141(64)$ & \\
Guardian depressive symptoms & $218(99)$ & $3.93(4.14) 0-21$ \\
Non cases & $182(83)$ & \\
Moderate & $19(9)$ & \\
Severe & $17(8)$ & \\
Guardian anxiety symptoms & \\
Non cases & $218(99)$ & $6.70(4.56) 0-21$ \\
Moderate & $132(60)$ & \\
Severe & $47(22)$ & \\
\hline
\end{tabular}

${ }^{a}$ Descriptives are provided here for which analysis was not carried out on. They merely provide further context

(80:20), but slightly higher than the ratio in other urban disadvantaged areas in the country (40:60) [53]. Parents, on average, had strong social support $(M=11.73, \mathrm{SD}=$ 2.34), which was higher than the average of a nonnationally representative sample of Irish adults in a randomised controlled trial (i.e. 10.70 or moderate social support) [48]; this was unexpected given that socioeconomically disadvantaged persons tend to have poor social support [54, 55]. Finally, parents had mean levels of depressive symptoms $(M=3.93, \mathrm{SD}=4.14)$ that fell within the normal range for general populations in the UK and the Netherlands [42, 43]. However, the $17 \%(N=36)$ of parents with moderate-to-severe symptoms is greater than the estimated prevalence of depressive disorders in general populations $[56,57]$ and similar to that in disadvantaged populations $[22,58]$.

\section{Association between BMI and HRQoL}

As expected, BMI was weakly inversely associated with total HRQoL $(r=-.15, p<.05)$, physical well-being $(r=-.17, p<.01)$, and autonomy and parent relations $(r=-.13, p<.05)$, and marginally inversely associated with psychological well-being $(r=-.12, p=.054)$ (Table 2). Statistically significant differences were seen only between those who were obese versus normal weight (on all aforementioned dimensions and total except for autonomy and parent relations). However, neither weight group fell below the average European HRQoL range.

Notably, none of the measured variables were found to confound the relationship between BMI and HRQoL (see supplementary Table 2). Other important (expected) bivariate associations included a weak negative correlation between age and school well-being and a weak positive correlation between age and 'autonomy and parent relations'. As expected, boys scored significantly higher than girls on 'social support and peer relations' and school wellbeing, but the magnitude of the differences in the means was small. Unexpectedly, parent's perceived social support and depressive symptoms, and family structure did not correlate with children's HRQoL. Finally, analysis revealed that BMI predicted physical well-being a year later (see Table 3) and vice versa, whilst autonomy and parent relations also predicted BMI a year later.

\section{Moderator effect of the BMI to HRQoL relationship}

Only the models testing the moderator effects of age, parent depressive symptoms and family structure in the relationship between BMI and HRQoL reached statistical significance and are presented here (child gender and parent perceived social support did not moderate the effect). In order to illustrate the interaction in Figs. 1, 2, 3, BMI, age and depressive symptoms were categorised into 'low' (1 SD below mean), 'medium' (mean) and 'high' (1 SD above mean). For example, low and medium BMI represents BMI at the lower and higher levels of the normal range, whilst high BMI represents overweight. The finding in Table 2 that for every point increase in BMI, HRQoL decreases by between .08 and .17 is also important to note when interpreting the figures.

Model 1: moderator effect of child age

The interaction between BMI and age made an independent contribution to children's physical $\left[\Delta R^{2}=.019, F_{\text {inc }}(1\right.$, $245)=5.09, p=.025]$ and school $\left[\Delta R^{2}=.038, F_{\text {inc }}(3\right.$, $245)=10.09, p=.002$ ] well-being. More specifically, under conditions of overweight status, children approaching adolescence (i.e. 12 years) reported significantly lower physical and school well-being than children aged between 7 and 11 years [see Fig. 1a and b, respectively]. Furthermore, the interaction between BMI and age made a marginal significant contribution to total HRQoL $\left[\Delta R^{2}=.016\right.$, $\left.F_{\text {inc }}(1,239)=3.857, p=.054\right]$ such that overweight children who were approaching adolescence reported marginally significantly lower total HRQoL than children aged 7-11 years (Fig. 1c). Total HRQoL, physical and school well-being did not differ by age when children were of normal weight.

Model 2: moderator effect of parent depressive symptoms

In addition, the interaction between child BMI and parent depressive symptoms made a significant contribution to children's school well-being $\left[\Delta R^{2}=.020, \quad F_{\text {inc }}(3\right.$, $244)=4.95, p=.027]$. In relation to 'overweight' 
Table 2 Relationship between BMI and HRQoL at year one (ANOVA and Pearson's correlation)

\begin{tabular}{|c|c|c|c|c|c|c|}
\hline \multirow[t]{2}{*}{ Independent variables } & \multicolumn{6}{|c|}{ Dimensions of and total HRQoL in children } \\
\hline & $\begin{array}{l}\text { Physical well- } \\
\text { being } \\
M(\mathrm{SD}) E S\end{array}$ & $\begin{array}{l}\text { Psychological } \\
\text { well-being } \\
M(\mathrm{SD}) E S\end{array}$ & $\begin{array}{l}\text { Autonomy \& } \\
\text { parent relations } \\
M(\mathrm{SD}) E S\end{array}$ & $\begin{array}{l}\text { Social Support \& } \\
\text { Peer Relations } \\
M(\mathrm{SD}) E S\end{array}$ & $\begin{array}{l}\text { School } \\
\text { Environment } \\
M(\mathrm{SD}) E S\end{array}$ & $\begin{array}{l}\text { Total } \\
\text { HRQoL } \\
M(\mathrm{SD}) E S\end{array}$ \\
\hline$B M I z$-scores $^{\mathrm{a}}$ & $-.17 * *$ & $-.12^{1}$ & $-.13 *$ & -.12 & -.08 & $-.15^{*}$ \\
\hline Normal/underweight (ref) & $55.07(11.28)$ & 54.98 (11.04) & $52.93(12.63)$ & $54.16(12.90)$ & $56.11(11.91)$ & $55.29(12.59)$ \\
\hline Overweight & $52.08(11.50)$ & $51.37(10.96)$ & $52.11(13.93)$ & $52.29(12.74)$ & $54.57(11.11)$ & $52.08(11.79)$ \\
\hline Obese & $50.51(9.48) .03 *$ & $51.20(10.45) .03^{\mathrm{b}}$ & $48.92(12.30)$ & $50.94(14.51)$ & $54.30(12.25)$ & $50.78(9.99) .03 *$ \\
\hline
\end{tabular}

ANOVA effect size (ES) is small at 0.01 , medium at 0.06 and large at 0.14

$* p<.05, * * p<.01$

${ }^{1} p=.054$

${ }^{a}$ Pearson's $r$ correlation ES is small when $r= \pm .10$ to \pm .29 ; medium when $r= \pm .30$ to \pm .49 ; and large when $r= \pm .50$ to \pm 1.0

b ANOVA found a small significant difference between groups but post hoc test did not identify any

Table 3 Pearson's $r$ correlation between BMI at year one and HRQoL in children at year two

\begin{tabular}{|c|c|c|c|c|c|c|}
\hline \multirow[t]{2}{*}{ Independent variables } & \multicolumn{6}{|c|}{ HRQoL in children at year two } \\
\hline & $\begin{array}{l}\text { Physical } \\
\text { well-being } \\
r\end{array}$ & $\begin{array}{l}\text { Psychological } \\
\text { well-being } \\
r\end{array}$ & $\begin{array}{l}\text { Autonomy \& } \\
\text { parent relations } \\
r\end{array}$ & $\begin{array}{l}\text { Social support \& } \\
\text { peer relations } \\
r\end{array}$ & $\begin{array}{l}\text { School } \\
\text { environment } \\
r\end{array}$ & $\begin{array}{l}\text { Total } \\
\text { HRQoL } \\
r\end{array}$ \\
\hline$B M I z$-scores at year one & $-.14 *$ & -.04 & -.04 & -.10 & .08 & -.10 \\
\hline
\end{tabular}

children, those whose parents had moderate-to-severe depressive symptoms as expected fared worse on school well-being than children whose parents were not depressed (Fig. 2). When children were of normal weight, school well-being did not differ between the different levels of parental depressive symptoms.

Model 3: moderator effect of family structure

Finally, the interaction between children's BMI and family structure made an independent contribution to their physical well-being $\left[\Delta R^{2}=.015, F_{\text {inc }}(1,247)=3.89, p=.05\right]$ and 'social support and peer relations' $\left[\Delta R^{2}=.035, F_{\text {inc }}(1\right.$, $245)=9.02, p<.003]$. Under conditions of overweight status, children from one-parent families reported significantly higher levels of physical well-being (Fig. 3a) and 'social support and peer relations' (Fig. 3(b)) than children from two-parent families. In contrast, when BMI was at the lower levels of the normal range, children from two-parent families reported significantly higher levels of physical well-being and 'social support and peer relations' than children of one-parent families. Physical well-being and 'social support and peer relations' did not differ by family structure when children had a BMI at the higher levels of the normal range.

\section{Discussion}

The results support the hypotheses that, in a sample of urban disadvantaged children, (1) BMI is weakly negatively correlated with HRQoL (weakly with 'total HRQoL', 'physical well-being', and 'autonomy and parent relations' and marginally with 'psychological well-being'); (2) the HRQoL of those categorised as obese is significantly worse than those of normal weight (on all aforementioned dimensions except for 'autonomy and parent relations'); and (3) BMI predicts physical well-being a year later and vice versa, whilst 'autonomy and parent relations' also predicts BMI a year later. The findings further support the hypotheses that the negative effect of high BMI on HRQoL intensifies as (4) children approach adolescence (12-13 years) and (5) as a parent's depressive symptoms become more severe (especially with respect to school and physical well-being and physical well-being, respectively).

The first two findings above are largely consistent with the HRQoL literature [8, 11, 59]. For example, Tsiros and colleagues (2009) in their meta-analysis reported that the risk to several dimensions of HRQoL increases as BMI increases and that HRQoL is significantly lower amongst those who were obese than those of normal weight, particularly with regards to total HRQoL and physical well- 

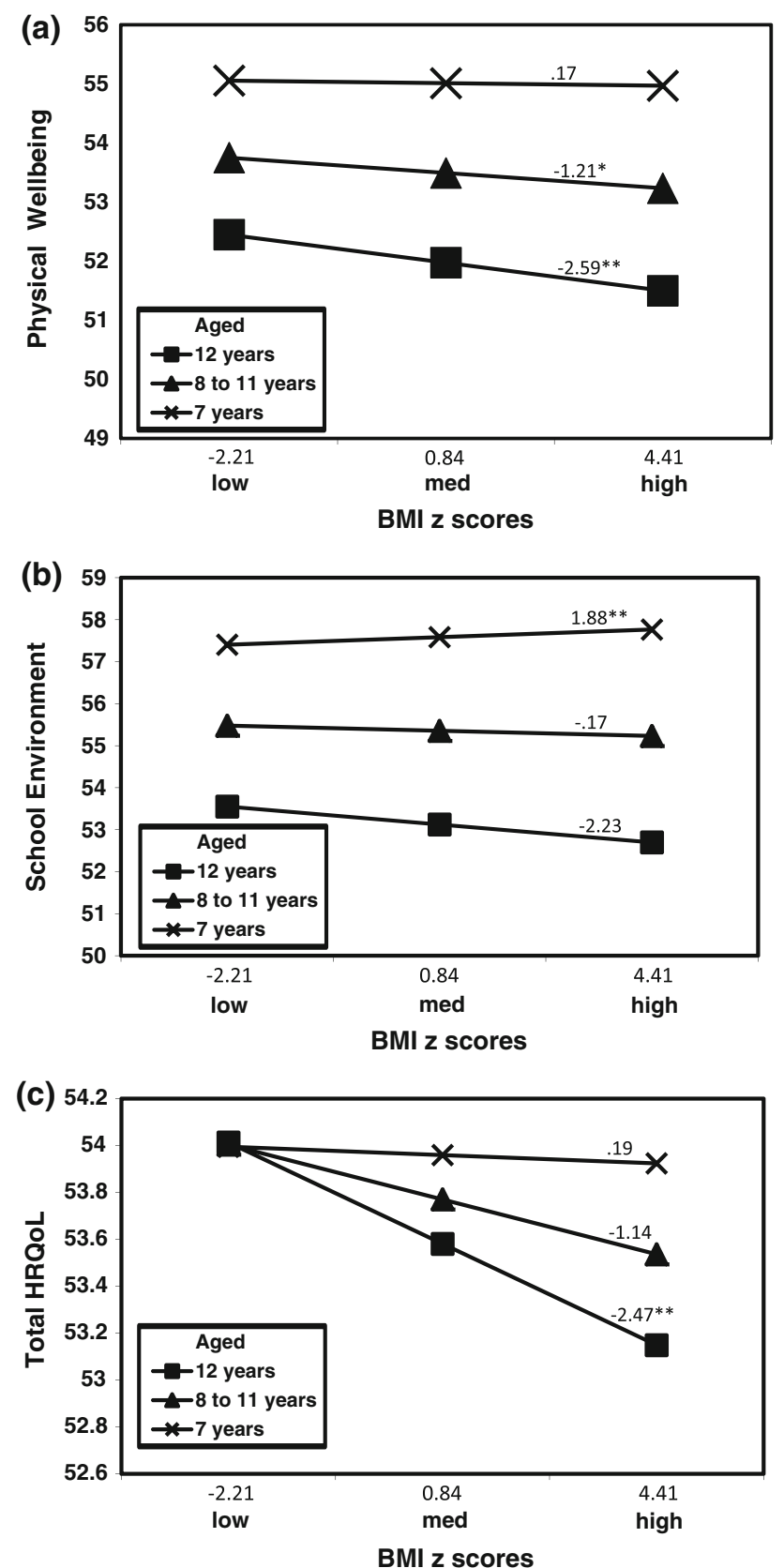

Fig. 1 a The relation between BMI z-scores and physical well-being for children aged 7, 8-11 and 12 years ( $T$ value indicates how slope differs from zero $(* * p<.01, * p<.05))$. b The relation between BMI $z$-scores and school environment for children aged 7, 8-11 and 12 years $[T$ value indicates how slope differs from zero $(* * p<.01)]$. c The relation between BMI $z$-scores and total HRQoL for children aged $7,8-11$ and 12 years [ $T$ value indicates how slope differs from zero $(* * p<.01)]$

being. Physical well-being may be affected because obesity is associated with a range of adverse physical consequences from dermatological to functional health problems [60]. The impairment to 'autonomy and parent relations' and even 'psychological well-being' may be due to the

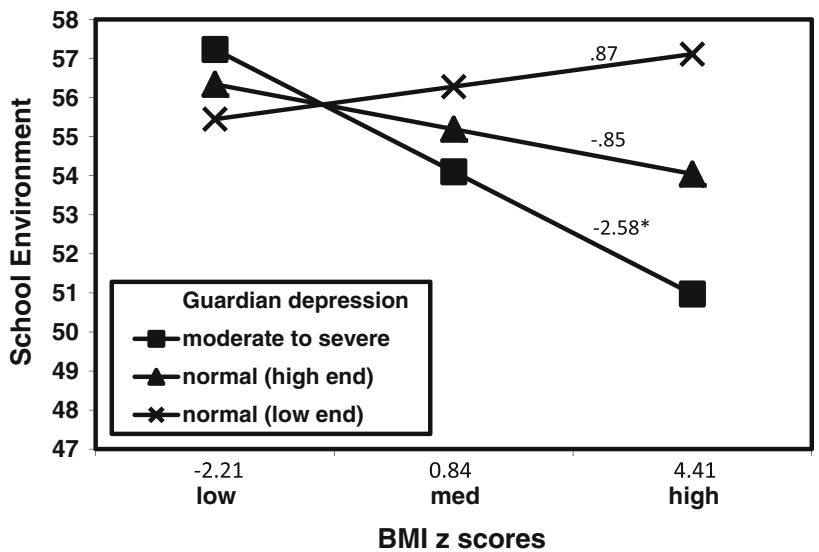

Fig. 2 The relation between BMI $z$-scores and school environment in children whose parent/guardian has normal and moderate-to-severe depressive symptoms [ $T$ value indicates how slope differs from zero $(* p<.05)]$

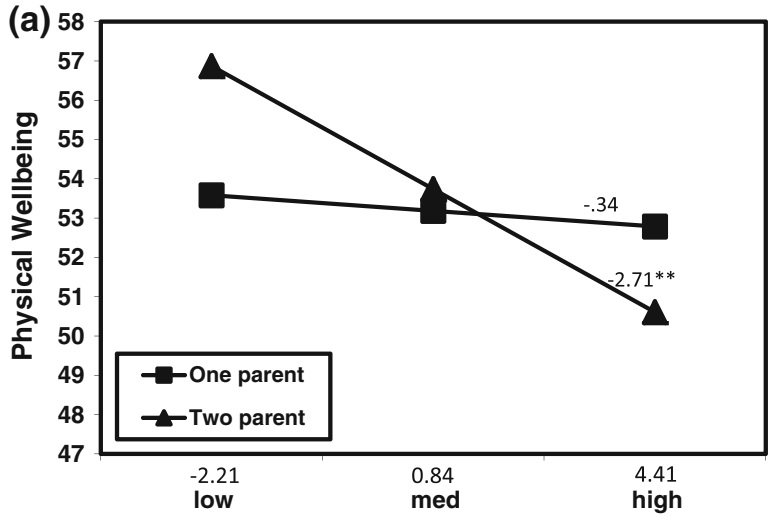

(b)

BMI z scores

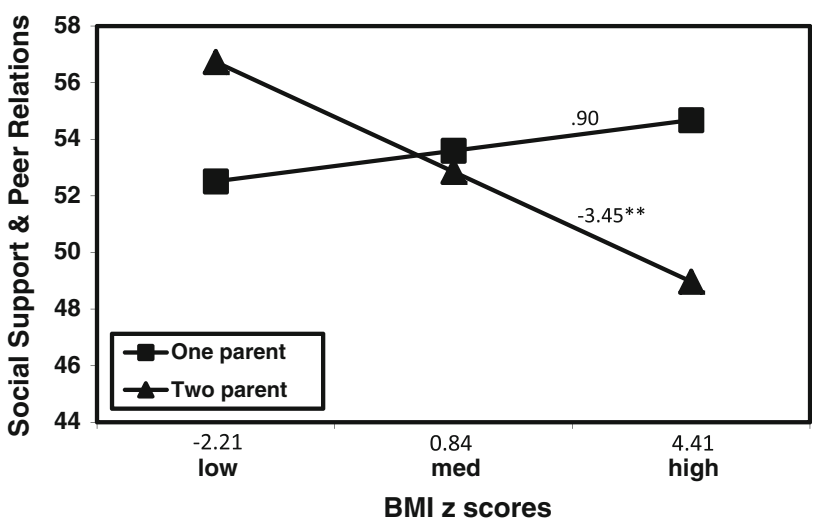

Fig. 3 a The relation between BMI $z$-scores and physical well-being for children from one-parent and two-parent families $[T$ value indicates how slope differs from zero $(* * p<.01)]$. b The relation between BMI $z$-scores and social support and peer relations for children from one-parent and two-parent families $[T$ value indicates how slope differs from zero $(* * p<.01)]$ 
psychosocial consequences of obesity such as body image concerns [61], weight-related teasing [62], low self-esteem, depression and interpersonal difficulties [63, 64]. Conversely, however-unlike numerous other studies [see 11] - there was no inverse association between children's BMI and their 'social support and peer relations'. Evidence from elsewhere to show that Irish children generally score above average on social support HRQoL [34] may explain why adiposity did not affect it; perhaps having a high BMI has little effect on friendships in Irish children, although this warrants further research attention.

It is noteworthy that the mean HRQoL for either weight group did not fall below the average European or Irish general population scores [34]. Typically, obese children have been found to have below average HRQoL scores although this is not always the case [24, 65]. As highlighted above, it was unexpected that the children in this study reported mean HRQoL scores within the published healthy range given that they are from low SES backgrounds. These findings may be explained by one or more factors including non-random sampling, social desirability responding, adaptation to deprived or overweight conditions, and/or a buffering effect as a result of these schools - amongst some of the most disadvantaged in the country-receiving additional health promoting resources (e.g. food provision programmes) as part of a Government scheme that is available only to schools designated as disadvantaged. However, further research is needed to properly explain these findings.

The third finding is somewhat consistent with the small number of longitudinal studies conducted in this area [e.g. $13 ; 14]$, which reported only weak evidence on the direction of causality between BMI and HRQoL in young people. For example, Herman and colleagues (2010) found significant negative correlations between BMI and a number of HRQoL dimensions during childhood, but young people's BMI only weakly predicted psychological well-being in adulthood. Similar to the first three findings reported here, small effect sizes are generally found in non-clinical studies investigating the effect of BMI on children's HRQoL $[8,11$, 59]. This may be because children with high BMI who have not been screened as overweight tend to be less aware of their overweight condition and are less likely to view it as an issue for their HRQoL than children who are receiving treatment for their condition [7, 66].

The fourth finding is not consistent with the small number of studies investigating the moderator effect of age in the relationship between BMI and HRQoL that reveal no significant interactions with respect to these HRQoL dimensions [24, 31, 67]. However, indirect evidence supports the fourth finding. For example, overweight children tend to report lower physical and school well-being than their non-overweight peers $[9,24,68]$, and, irrespective of weight, children report poorer physical and school wellbeing as they get older [69-71]. These findings may be due to several puberty-related factors including greater changes to body composition [72], increased societal pressures to be thin [73] and greater susceptibility to depression and anxiety [74]. Notably, girls are more vulnerable than boys to the last two of these [74, 75].

Although up until now, there have been no studies investigating the moderator effect identified in this study (i.e. the fifth finding), it has been shown that depression in parents of overweight children can lead to parenting stress [76] which, in turn, has been found to intensify the negative effect of being overweight on children's school well-being $[77,78]$. Further indirect evidence is that children whose parents are depressed are at a greater risk of being overweight [25, 26, 79] and of having poor school well-being $[27,80]$ when compared to children whose parents are not depressed, and that overweight BMI is inversely associated with children's school well-being [24, 68]. These relationships may exist because depressed parents with overweight children are more likely to engage in a parenting style that sets few household rules and responsibilities for children, and does not monitor children's healthy food choices, exercise or schooling [81, 82].

Unexpectedly, the hypotheses that the negative effect of elevated BMI on children's HRQoL is intensified for females and diminished for children whose parents have strong social support (or who live in two-parent families) were not supported by this study. Perhaps significant interactions may only be identified when the age and gender of the child are considered simultaneously; significant differences between genders in HRQoL are usually not seen until children reach the age of 12 [71, 83]. In terms of parent's social support, significant interactions may not have been observed because social support may only serve to protect parents from stressors and not children. Instead, the social support provided to children by their parents may be a moderator. The unexpected buffering effect of one-parent families on the 'physical wellbeing' and 'social support and peer relations' of those children with overweight status is difficult to explain. It may be because children living in greater disadvantage (e.g. one-parent families) are less likely to perceive themselves as overweight [84] and to negatively evaluate these dimensions. Alternatively, the identification of a greater proportion than expected of two-parent families and parents with strong social support suggests a potential source of bias in both the sampling and the results although it is difficult to be clear on this without further research.

The findings reported here represent an important addition to the literature in a number of ways. It addressed an important gap in knowledge and theory relating to the association between children's BMI and HRQoL by 
extending the research into moderators of this relationship. It also involved a moderate sample of young urban disadvantaged children $(N=255)$, a cohort under-represented in the HRQoL literature. The use of self-report measures with good psychometric properties and the assessment of children's BMI by trained nurses helped to ensure that results were reliable and could be generalised to other urban disadvantaged populations. The use of a generic HRQoL measure further enhanced applicability to all population subgroups (e.g. obese or disadvantaged) [85]. Furthermore, this study generally used measures that generated continuous data and more can be learned from analysing such data [86]. Finally, the incorporation of a longitudinal element strengthened the research design and allowed for an exploration of causal relationships [87].

However, several limitations of the study should be considered when interpreting the findings. Firstly, participants were recruited using a non-randomised sampling method which may have increased the risk of selection bias [88] and caution should be taken, therefore, when generalising the results to populations other than those living in urban disadvantage. Secondly, Kidscreen was administered to children aged $7(N=21)$ despite not being psychometrically tested for children under 8 [34]. However, research shows that these children can reliably report on their HRQoL (see [89]) and several studies on the HRQoL of children aged 7 have been published (e.g. [90]). Thirdly, whilst some HRQoL distributions were skewed and nonnormal, this study followed the tradition within HRQoL research of using parametric tests [91, 92] whilst confirming the robustness of these with nonparametric tests.

The findings from this study have important implications for policy and practice. With greater than expected prevalence of overweight and obesity, governments may need to prioritise urban disadvantaged children in their policy making $[93,94]$. Perhaps the accessibility of food, the built environment or perceptions of safety of urban disadvantaged areas lead to poorer diets and more sedentary lifestyles [95]. Furthermore, preventions, interventions and treatments which aim to prevent or reduce obesity, traditionally by encouraging physical activity and healthy eating [96], may also need to aim to optimise the HRQoL of urban disadvantaged preadolescents. If, however, weight management methods fail to improve HRQoL, obesity programmes may have to intervene at the HRQoL level. For example, given that obese children have poorer, albeit not clinically poor, 'physical well-being' and 'autonomy and parent relations' than normal weight children, these programmes could aim to ensure easy mobility around the home and school, address any physical health and sleep complaints, provide opportunities for children to create their own social and leisure time, and improve parent-child interactions.
Obesity interventions and treatments that aim to improve HRQoL of overweight children may also need to prevent or treat parent's mental ill health possibly through the incorporation of parenting training, an approach that has proven to be effective at improving parenting skills, parent mental ill health and health outcomes for children including achieving and maintaining a healthy weight $[97$, 98]. Obesity programmes could also aim, for example, to improve children's perception of school and their abilities therein. Moreover, the findings of this study imply that obesity programmes may need to monitor children's HRQoL to help design or adjust programmes to improve aspects of life which really matter to a child and to determine their effectiveness [89].

This study could be replicated in the future, but with some adjustments to sampling, design, measures and analysis. These might include the use of random sampling of children to increase the generalizability of the findings to other urban disadvantaged populations and to children more generally. It might also be useful to include more fathers in view of the gender differences in the effect of paternal depression on children's health (see [99] ). In addition, findings could be compared between studies that use different measures of HRQoL based on different constructs. Furthermore, the levels of social support actually received by parents and/or children should be measured in order to obtain a more accurate representation of this important variable. As BMI explained only a small amount of the variance in HRQoL, greater attention may need to be given to the process by which adiposity is influential in this regard. For example, research suggests that body image concerns or weight-related teasing may be the mechanisms by which obesity affects HRQoL [59]. To determine whether obesity causes poor HRQoL, further longitudinal analysis could also be conducted. Finally, the aspects of disadvantaged urban areas that may lead to greater levels of overweight and obesity than in disadvantaged populations more generally could also be explored.

In summary, an increasing BMI was associated with a decreasing HRQoL in urban disadvantaged children and when BMI reached overweight, children approaching adolescence and whose parents had high levels of depressive symptoms fared worse on HRQoL than younger children whose parents were not depressed. Obesity programmes could aim to prevent/reduce obesity and optimise HRQoL in urban disadvantaged preadolescent children whilst also targeting parental mental health difficulties. Future research should attempt to delineate the mechanism by which adiposity affects HRQoL.

Acknowledgments This research was funded by the Irish Research Council and the Childhood Development Initiative which is co-funded by the Atlantic Philanthropies and the Department of Children 
and Youth Affairs. We are extremely grateful to all the parents, guardians, children and school staff for taking part in, and facilitating, this study.

\section{References}

1. Wijnhoven, T., Raaij, J., Spinelli, A., Rito, A., Hovengen, R., Kunesova, M., et al. (2012). WHO European Childhood Obesity Surveillance Initiative 2008: weight, height and body mass index in 6-9-year-old children. Pediatric Obesity.

2. Whelton, H., Harrington, J., Crowley, E., Kelleher, V., Cronin, M., \& Perry, I. J. (2007). Prevalence of overweight and obesity on the island of Ireland: results from the North South Survey of Children's Height, Weight and Body Mass Index, 2002. BMC Public Health, 7(1), 187.

3. Dietz, W. H. (1998). Health consequences of obesity in youth: Childhood predictors of adult disease. Pediatrics, 101(Supplement 2), 518-525.

4. Russell-Mayhew, S., McVey, G., Bardick, A., \& Ireland, A. (2012). Mental health, wellness, and childhood overweight/ obesity. Journal of Obesity, 2012, 9.

5. Taylor, R. M., Gibson, F., \& Franck, L. S. (2008). A concept analysis of health-related quality of life in young people with chronic illness. Journal of Clinical Nursing, 17(14), 1823-1833.

6. Bronfenbrenner, U. (2005). Making humans beings human. London: Sage Publications.

7. Ferrans, C. E., Zerwic, J. J., Wilbur, J. E., \& Larson, J. L. (2005). Conceptual model of health-related quality of life. Journal of Nursing Scholarship, 37(4), 336-342.

8. Ottova, V., Erhart, M., Rajmil, L., Dettenborn-Betz, L., \& Ravens-Sieberer, U. (2012). Overweight and its impact on the health-related quality of life in children and adolescents: Results from the European KIDSCREEN survey. Quality of Life Research, 21(1), 59-69.

9. Schwimmer, J. B., Burwinkle, T. M., \& Varni, J. W. (2003). Health-related quality of life of severely obese children and adolescents. JAMA, the Journal of the American Medical Association, 289(14), 1813-1819.

10. Varni, J., Limbers, C., \& Burwinkle, T. (2007). Impaired healthrelated quality of life in children and adolescents with chronic conditions: A comparative analysis of 10 disease clusters and 33 disease categories/severities utilizing the PedsQLTM 4.0 Generic Core Scales. Health and Quality of Life Outcomes, 5(1), 43.

11. Tsiros, M. D., Olds, T., Buckley, J. D., Grimshaw, P., Brennan, L., Walkley, J., et al. (2009). Health-related quality of life in obese children and adolescents. International Journal of Obesity, 33(4), 387-400.

12. Morales, P. F., Sánchez-López, M., Moya-Martínez, P., GarcíaPrieto, J. C., Martínez-Andrés, M., García, N. L., \& MartínezVizcaíno, V. (2012). Health-related quality of life, obesity, and fitness in schoolchildren: the Cuenca study. Quality of Life Research, 1-9.

13. Herman, K. M., Hopman, W. M., \& Craig, C. L. (2010). Are youth BMI and physical activity associated with better or worse than expected health-related quality of life in adulthood? The physical activity longitudinal study. Quality of Life Research, 19(3), 339-349.

14. Williams, J. W., Canterford, L., Hesketh, K. D., Hardy, P., Waters, E. B., Patton, G. C., et al. (2011). Changes in body mass index and health related quality of life from childhood to adolescence. International Journal of Pediatric Obesity, 6(2Part2), e442-e448.
15. Hollywood, E., Comiskey, C., Begley, T., Snel, A., O'Sullivan, K., Quirke, M., \& Wynne, C. (2012). Measuring and modelling body mass index among a cohort of urban children living with disadvantage. Journal of Advanced Nursing.

16. Knai, C., Lobstein, T., Darmon, N., Rutter, H., \& McKee, M. (2012). Socioeconomic patterning of childhood overweight status in Europe. International Journal of Environmental Research and Public Health, 9(4), 1472-1489.

17. Von Rueden, U., Gosch, A., Rajmil, L., Bisegger, C., \& RavensSieberer, U. (2006). Socioeconomic determinants of health related quality of life in childhood and adolescence: Results from a European study. Journal of Epidemiology and Community Health, 60(2), 130.

18. Conger, R., \& Elder, G. H. (1994). Families in troubled times: Adapting to change in rural America: Aldine de Gruyter.

19. Becker, G. S., \& Tomes, N. (1994). Human capital and the rise and fall of families. In Human capital: A theoretical and empirical analysis with special reference to education (3rd Ed) (pp. 257-298): The University of Chicago Press.

20. Blau, D. M. (1999). The effect of income on child development. Review of Economics and Statistics, 81(2), 261-276.

21. Reading, R., \& Reynolds, S. (2001). Debt, social disadvantage and maternal depression. Social Science and Medicine, 53(4), 441-453.

22. Kiernan, K. E., \& Huerta, M. C. (2008). Economic deprivation, maternal depression, parenting and children's cognitive and emotional development in early childhood. British Journal of Sociology, 59(4), 783-806.

23. Hughes, A. R., Farewell, K., Harris, D., \& Reilly, J. J. (2007). Quality of life in a clinical sample of obese children. International Journal of Obesity, 31(1), 39-44.

24. Pinhas-Hamiel, O., Singer, S., Pilpel, N., Fradkin, A., Modan, D., \& Reichman, B. (2005). Health-related quality of life among children and adolescents: Associations with obesity. International Journal of Obesity, 30(2), 267-272.

25. Favaro, A., \& Santonastaso, P. (1995). Effects of parents' psychological characteristics and eating behaviour on childhood obesity and dietary compliance. Journal of Psychosomatic Research, 39(2), 145-151.

26. Zipper, E., Vila, G., Dabbas, M., Bertrand, C., Mouren-Simeoni, M. C., Robert, J. J., et al. (2001). Obesity in children and adolescents, mental disorders and familial psychopathology (pp. 755-4982) (print).

27. Wiegand-Grefe, S., Halverscheid, S., Petermann, F., \& Plass, A. (2012). Psychopathology and quality of life in children of mentally ill parents.

28. Gerald, L. B., Anderson, A., Johnson, G. D., Hoff, C., \& Trimm, R. F. (1994). Social class, social support and obesity risk in children. Child: Care, Health and Development, 20(3), 145-163.

29. Gibson, L. Y., Byrne, S. M., Davis, E. A., Blair, E., Jacoby, P., \& Zubrick, S. R. (2007). The role of family and maternal factors in childhood obesity. Medical Journal of Australia, 186(11), 591.

30. Bastiaansen, D., Koot, H. M., \& Ferdinand, R. F. (2005). Psychopathology in children: Improvement of quality of life without psychiatric symptom reduction? European Child and Adolescent Psychiatry, 14(7), 364-370.

31. Swallen, K., Reither, E., Haas, S., \& Meier, A. (2005). Overweight, obesity, and health-related quality of life among adolescents: The National Longitudinal Study of Adolescent Health. Pediatrics, 115(2), 340.

32. Department of Education and Science. (2003). Delivering equality of opportunity in schools. Dublin: Government Publications.

33. Haase, T., \& Pratschke, J. (2008). New measures of deprivation for the Republic of Ireland. Dublin: Pobal. 
34. Kidscreen Group Europe. (2006). The Kidscreen questionnaires. Quality of life questionnaires for children and adolescentshandbook. Lengerich: Papst Science Publisher.

35. Robitail, S., Ravens-Sieberer, U., Simeoni, M. C., Rajmil, L., Bruil, J., Power, M., et al. (2007). Testing the structural and cross-cultural validity of the KIDSCREEN-27 quality of life questionnaire. Quality of Life Research, 16(8), 1335-1345.

36. Ravens-Sieberer, U., Auquier, P., Erhart, M., Gosch, A., Rajmil, L., Bruil, J., et al. (2007). The KIDSCREEN-27 quality of life measure for children and adolescents: psychometric results from a cross-cultural survey in 13 European countries. Quality of Life Research, 16(8), 1347-1356.

37. WHO. (2009). WHO AnthroPlus for personal computers manual: software for assessing growth of the world's children and adolescents. Geneva: World Health Organization.

38. Onis, M., Onyango, A. W., Borghi, E., Siyam, A., Nishida, C., \& Siekmann, J. (2007). Development of a WHO growth reference for school-aged children and adolescents. Bulletin of the World Health Organization, 85(9), 660-667.

39. Zigmond, A. S., \& Snaith, R. (1983). The hospital anxiety and depression scale. Acta Psychiatrica Scandinavica, 67(6), 361-370.

40. Bjelland, I., Dahl, A. A., Haug, T. T., \& Neckelmann, D. (2002). The validity of the Hospital Anxiety and Depression Scale-An updated literature review. Journal of Psychosomatic Research, 52(2), 69-78.

41. Herrmann, C. (1997). International experiences with the Hospital Anxiety and Depression Scale-a review of validation data and clinical results. Journal of Psychosomatic Research, 42(1), $17-41$.

42. Crawford, J., Henry, J., Crombie, C., \& Taylor, E. (2001). Normative data for the HADS from a large non-clinical sample. British Journal of Clinical Psychology, 40(4), 429-434.

43. Spinhoven, P., Ormel, J., Sloekers, P., Kempen, G., Speckens, A., \& Hemert, A. V. (1997). A validation study of the Hospital Anxiety and Depression Scale (HADS) in different groups of Dutch subjects. Psychological Medicine, 27(2), 363-370.

44. Snaith, P. (1994). The hospital anxiety and depression scale with The irritability-depression-anxiety scale and the leeds situational anxiety scale manual: NFER-Nelson.

45. Lisspers, J., Nygren, A., \& Söderman, E. (1997). Hospital Anxiety and Depression Scale (HAD): Some psychometric data for a Swedish sample. Acta Psychiatrica Scandinavica, 96(4), 281-286.

46. Mykletun, A., Stordal, E., \& Dahl, A. A. (2001). Hospital Anxiety and Depression (HAD) scale: Factor structure, item analyses and internal consistency in a large population. The British Journal of Psychiatry, 179(6), 540-544.

47. Meltzer, H. (2003). Development of a common instrument for mental health. In A. Nosikov \& C. Gudex (Eds.), EUROHIS: Developing common instruments for health surveys (pp. 35-60). Amsterdam: IOS Press.

48. Dalgard, O. (2006). Sosiale risikofaktorer, psykisk helse og forebyggende arbeid. Folkehelseinstituttet: Oslo.

49. Aiken, L., \& West, S. (1991). Testing and interpreting interactions in multiple regression: Newbury Park. CA: Sage.

50. Cohen, J. (1992). A power primer. Psychological Bulletin, $112(1), 155$.

51. Keenaghan, C., \& Kilroe, J. (2008). A study on the quality of life tool KIDSCREEN for children and adolescents in Ireland: Results of the KIDSCREEN National Survey 2005.

52. Layte, R., \& McCrory, C. (2011). Growing up in Ireland: National longitudinal study of children: Overweight and obesity among 9-year-olds: Stationery office.

53. McKeown, K., Haase, T., \& Pratschke, J. (2003). Family wellbeing: What makes a difference? The Ceifin Centre and Dept. of Social and Family Affairs.
54. Krause, N., \& Borawski-Clark, E. (1995). Social class differences in social support among older adults. The Gerontologist, 35(4), 498-508.

55. Weyers, S., Dragano, N., Möbus, S., Beck, E.-M., Stang, A., Möhlenkamp, S., et al. (2010). Poor social relations and adverse health behaviour: Stronger associations in low socioeconomic groups? International Journal of Public Health, 55(1), 17-23.

56. O'Hara, M. W., \& Swain, A. M. (1996). Rates and risk of postpartum depression-a meta-analysis. International review of psychiatry, 8(1), 37-54.

57. Wittchen, H.-U., \& Jacobi, F. (2005). Size and burden of mental disorders in Europe-a critical review and appraisal of 27 studies. European Neuropsychopharmacology, 15(4), 357-376.

58. Olfson, M., Shea, S., Feder, A., Fuentes, M., Nomura, Y., Gameroff, M., et al. (2000). Prevalence of anxiety, depression, and substance use disorders in an urban general medicine practice. Archives of Family Medicine, 9(9), 876.

59. Haraldstad, K., Christophersen, K.-A., Eide, H., Nativg, G. K., \& Helseth, S. (2011). Predictors of health-related quality of life in a sample of children and adolescents: A school survey. Journal of Clinical Nursing, 20(21-22), 3048-3056.

60. Wabitsch, M. (2000). Overweight and obesity in European children: Definition and diagnostic procedures, risk factors and consequences for later health outcome. European Journal of Pediatrics, 159(13), 8-13.

61. Wardle, J., \& Cooke, L. (2005). The impact of obesity on psychological well-being. Best Practice \& Research Clinical Endocrinology \& Metabolism, 19(3), 421-440.

62. Puhl, R. M., \& Latner, J. D. (2007). Stigma, obesity, and the health of the nation's children. Psychological Bulletin, 133(4), 557.

63. Datar, A., \& Sturm, R. (2006). Childhood overweight and elementary school outcomes. International Journal of Obesity, 30(9), 1449-1460.

64. Latner, J. D., \& Stunkard, A. J. (2003). Getting worse: The stigmatization of obese children. Obesity Research, 11(3), 452-456.

65. Zeller, M. H., \& Modi, A. C. (2006). Predictors of health-related quality of life in obese youth. Obesity, 14(1), 122-130.

66. Cummins, R. A., Gullone, E., \& Lau, A. L. D. (2003). A model of subjective well-being homeostasis: The role of personality. The universality of subjective wellbeing indicators, pp. 7-46.

67. Hughes, A. R., Farewell, K., Harris, D., \& Reilly, J. J. (2006). Quality of life in a clinical sample of obese children. International Journal of Obesity, 31(1), 39-44.

68. Zeller, M. H., Roehrig, H. R., Modi, A. C., Daniels, S. R., \& Inge, T. H. (2006). Health-related quality of life and depressive symptoms in adolescents with extreme obesity presenting for bariatric surgery. Pediatrics, 117(4), 1155-1161.

69. Bisegger, C., Cloetta, B., von Bisegger, U., Abel, T., \& RavensSieberer, U. (2005). Health-related quality of life: Gender differences in childhood and adolescence. Sozial-und Präventivmedizin/Social and Preventive Medicine, 50(5), 281-291.

70. Michel, G., Bisegger, C., Fuhr, D., \& Abel, T. (2009). Age and gender differences in health-related quality of life of children and adolescents in Europe: A multilevel analysis. Quality of Life Research, 18(9), 1147-1157.

71. Palacio-Vieira, J., Villalonga-Olives, E., Valderas, J., Espallargues, M., Herdman, M., Berra, S., et al. (2008). Changes in health-related quality of life (HRQoL) in a population-based sample of children and adolescents after 3 years of follow-up. Quality of Life Research, 17(10), 1207-1215.

72. Malina, R. M., Bouchard, C., \& Bar-Or, O. (2004). Growth, maturation, and physical activity. Champaign: Human Kinetics Publishers. 
73. Blowers, L. C., Loxton, N. J., Grady-Flesser, M., Occhipinti, S., \& Dawe, S. (2003). The relationship between sociocultural pressure to be thin and body dissatisfaction in preadolescent girls. Eating Behaviors, 4(3), 229-244.

74. Parker, G., \& Brotchie, H. (2010). Gender differences in depression. International Review of Psychiatry, 22(5), 429-436.

75. Presnell, K., Bearman, S. K., \& Stice, E. (2004). Risk factors for body dissatisfaction in adolescent boys and girls: A prospective study. International Journal of Eating Disorders, 36(4), 389-401.

76. Moens, E., Braet, C., Bosmans, G., \& Rosseel, Y. (2009). Unfavourable family characteristics and their associations with childhood obesity: A cross-sectional study. European Eating Disorders Review, 17(4), 315-323.

77. Guilfoyle, S. M., Zeller, M. H., \& Modi, A. C. (2010). Parenting stress impacts obesity-specific health-related quality of life in a pediatric obesity treatment-seeking sample. Journal of Developmental and Behavioral Pediatrics: JDBP, 31(1), 17.

78. Janicke, D. M., Marciel, K. K., Ingerski, L. M., Novoa, W., Lowry, K. W., Sallinen, B. J., et al. (2007). Impact of psychosocial factors on quality of life in overweight youth. Obesity, 15(7), 1799-1807.

79. Epstein, L. H., Myers, M. D., \& Anderson, K. (1996). The association of maternal psychopathology and family socioeconomic status with psychological problems in obese children. Obesity Research, 4(1), 65-74.

80. Wiegand-Grefe, S., Jeske, J., Bullinger, M., Plaß, A., \& Petermann, F. (2010). Lebensqualität von Kindern psychisch kranker Eltern. Zeitschrift für Psychiatrie, Psychologie und Psychotherapie, 58(4), 315-322.

81. Sleddens, E. F. C., Gerards, S. M. P. L., Thijs, C., Vries, N. K., \& Kremers, S. P. J. (2012). General parenting, childhood overweight and obesity-inducing behaviors: A review. International journal of pediatric obesity, 6(2Part2), e12-e27.

82. Topham, G. L., Page, M. C., Hubbs-Tait, L., Rutledge, J. M., Kennedy, T. S., Shriver, L., et al. (2010). Maternal depression and socio-economic status moderate the parenting style/child obesity association. Public Health Nutrition, 13(8), 1237-1244.

83. Benjet, C., \& Hernández-Guzmán, L. (2002). A short-term longitudinal study of pubertal change, gender, and psychological well-being of Mexican early adolescents. Journal of Youth and Adolescence, 31(6), 429-442.

84. O'Dea, J. A., \& Caputi, P. (2001). Association between socioeconomic status, weight, age and gender, and the body image and weight control practices of 6-to 19-year-old children and adolescents. Health Education Research, 16(5), 521-532.

85. Robitail, S., Ravens-Sieberer, U., Simeoni, M.-C., Rajmil, L., Bruil, J., Power, M., et al. (2007). Testing the structural and cross-cultural validity of the KIDSCREEN-27 quality of life questionnaire. Quality of Life Research, 16(8), 1335-1345.

86. Turner, E. L., Dobson, J. E., \& Pocock, S. J. (2010). Categorisation of continuous risk factors in epidemiological publications: a survey of current practice. Epidemiologic Perspectives \& Innovations, 7(1), 9.
87. Page, R. M., Cole, G. E., \& Timmreck, T. C. (1995). Basic epidemiological method \& biostat: A practical guidebook: Jones \& Bartlett learning.

88. Berra, S., Ravens-Sieberer, U., Erhart, M., Tebe, C., Bisegger, C., Duer, W., et al. (2007). Methods and representativeness of a European survey in children and adolescents: the KIDSCREEN study. BMC Public Health, 7(1), 182.

89. Matza, L., Swensen, A., Flood, E., Secnik, K., \& Leidy, N. (2004). Assessment of health-related quality of life in children: A review of conceptual, methodological, and regulatory issues. Value in Health, 7, 79-92.

90. Hijmans, C., Fijnvandraat, K., Oosterlaan, J., Heijboer, H., Peters, M., \& Grootenhuis, M. (2010). Double disadvantage: A case control study on health-related quality of life in children with sickle cell disease. Health and Quality of Life Outcomes, 8(1), 121.

91. Giannakopoulos, G., Dimitrakaki, C., Pedeli, X., Kolaitis, G., Rotsika, V., Ravens-Sieberer, U., et al. (2009). Adolescents' wellbeing and functioning: Relationships with parents' subjective general physical and mental health. Health and Quality of Life Outcomes, 7(1), 100.

92. Ravens-Sieberer, U., Gosch, A., Rajmil, L., Erhart, M., Bruil, J., Duer, W., et al. (2005). KIDSCREEN-52 quality-of-life measure for children and adolescents. Expert Review of Pharmacoeconomics \& Outcomes Research, 5(3), 353-364.

93. Commission, European. (2007). White paper on a strategy for Europe on nutrition. Overweight and Obesity related health issues: OOPEC.

94. Health Services Executive. (2010). Health services executive framework for action on obesity 2008-2012. Government of Ireland.

95. Foster, S., \& Giles-Corti, B. (2008). The built environment, neighborhood crime and constrained physical activity: An exploration of inconsistent findings. Preventive Medicine, 47(3), 241-251.

96. Friedman, R. R., \& Schwartz, M. B. (2008). Public policy to prevent childhood obesity, and the role of pediatric endocrinologists. Journal of Pediatric Endocrinology and Metabolism, 21(8), 717-726.

97. Golan, M., Kaufman, V., \& Shahar, D. R. (2006). Childhood obesity treatment: Targeting parents exclusively v. parents and children. British Journal of Nutrition, 95(5), 1008-1015.

98. McGilloway, S., Mhaille, G. N., Bywater, T., Furlong, M., Leckey, Y., Kelly, P., et al. (2012). A parenting intervention for childhood behavioral problems: A randomized controlled trial in disadvantaged community-based settings. Journal of Consulting and Clinical Psychology, 80(1), 116-127.

99. Connell, A. M., \& Goodman, S. H. (2002). The association between psychopathology in fathers versus mothers and children's internalizing and externalizing behavior problems: A meta-analysis. Psychological Bulletin, 128(5), 746. 